\title{
Assessment of High-lift Concepts for a Regional Aircraft in the ALONOCO Project
}

\author{
Peter Eliasson, Olof Grundestam and Shia-Hui Peng \\ FOI, Swedish Defence Research Agency, SE-16490 Stockholm, Sweden \\ Huadong Yao, Lars Davidson and Lars-Erik Eriksson \\ Chalmers University of Technology, SE-412 96, Gothenburg, Sweden
}

\begin{abstract}
This work introduces the work conducted in the EU JTI project ANOLOCO, which has aimed at an assessment of aerodynamic and aeroacoustic performance of several high-lift configurations of a regional aircraft. The high-lift designs are for a laminar and slat-less wing, including configurations with a double slotted flap, single slotted flap, drooped nose and a Krueger flap. The aerodynamic performance is assessed from steady state RANS calculations up to maximum lift. The aeroacoustic performance is based on hybrid RANSLES calculations for flow-induced noise generation, and using acoustic analogy methods for far-field noise propagation. Three different analogy methods are evaluated and compared. The assessment shows that the configuration with a Krueger flap gives the best performance. The maximum lift is close to $20 \%$ higher than for any other configuration and the noise levels are also reduced, up to $10 \mathrm{~dB}$ lower than the configuration with a double slotted flap.
\end{abstract}

\section{Introduction}

The next generation of commercial transport aircraft is confronted with challenging targets in terms of reduced fuel consumption and emissions as well as more strict noise regulation requirements. To enhance the efficiency at cruise flight with reduced drag, the wing can be designed such that a major part of the wing is kept laminar. This can be achieved by a wing design based on Natural Laminar Flow (NLF) where instabilities causing laminar to turbulent transition are suppressed to occur as far downstream as possible on the wing. ${ }^{1,2}$ The laminar wing has implications on the high-lift system since a forward slat element is avoided due to the introduction of glitches and steps when retracted that usually introduces disturbances that destroys the laminar flow. Alternative high-lift configurations are then considered, e.g. a droop nose or a Krueger flap deployed from the lower side of the wing. These high-lift systems are typically less efficient than conventional systems with a reduced maximum lift. It is hence crucial to select a system which has a good performance and thus as high maximum lift as possible.

High-lift devices are significant contributors to aircraft external noise for conventional aircraft. ${ }^{3,4}$ Future aircraft development will therefore need to incorporate measures to reduce noise levels at landing and take-off. Although the high-lift configurations of laminar wings do not contain a conventional forward slat which is known as one of the dominating noise sources, ${ }^{5,6}$ there are other significant sources of noise such as e.g. slotted flaps. Computational Aero Acoustic (CAA) methods can provide detailed understanding of flow-generated noise. A reliable use of CAA relies however on turbulence-resolving simulations to incorporate turbulent fluctuations into noise-generating sources. Hybrid RANS-LES computations have been increasingly used in aerodynamic applications and have shown promising capabilities ${ }^{7-9}$. Their applications in analysis of flow-induced noise sources have also been increased over the recent years. ${ }^{10-13}$ The prediction of the noise of an entire aircraft is still extremely expensive due to the required high resolution of the aircraft and its components in both the computational grid as well as in time to resolve relevant scales contributing to the noise.

The investigation presented in this paper is a result of an assessment of four different low-noise high-lift concepts for a regional aircraft with a laminar wing. The work has been carried out in an EU sponsored project called ALONOCO in the program of the Clean Sky Joint Undertaking (CSJU). The project is part of a Low-Noise Configuration project under the Green Regional Aircraft (GRA) Integrated Technology Demonstrator for which Alenia Aeronautica is responsible and who has provided the flow and geometric details of the configuration for assessment. ALONOCO is a 18 -month project with two Swedish partners, FOI and Chalmers.

The objective of the work has been to investigate the aerodynamic performance and noise emissions from four high-lift landing configurations. The underlying wing is a wing designed for NLF and has been designed within the 
Clean Sky GRA. Since the wing is designed for laminar flow, the considered high-lift concepts do not contain a conventional forward slat. Instead, the high-lift configurations are based on different concepts of the flap, the wing nose and a Krueger flap. These concepts were also designed within the Clean Sky GRA consortium. The selected concepts have been investigated and assessed in three dimensions in ALONOCO. Five different concepts are evaluated in total including a double slotted flap as initial baseline configuration, a single slotted flap, two droop nose configurations with single slotted flap and finally a configuration with a forward Krueger flap and a single slotted flap.

The assessment is based on CFD calculations where the aerodynamic assessment is carried out with steady state RANS calculations. The assessment is mainly based on maximum lift predictions to determine if some of the concepts have a higher maximum lift level than the other ones. The aeroacoustic assessment is based on unsteady hybrid RANS-LES calculations for three of the high-lift configurations from which unsteady data is used as input to acoustic analogy methods to predict the noise perceived of an observer at far field. The noise levels as well as frequency contents for the three analyzed configurations are compared, the three acoustic analogy methods are also compared and one of the methods is used to separate the noise from different aircraft components to determine the dominating noise source.

This paper summarizes all results obtained in the ALONCO project. Separate papers with more details of the aerodynamic ${ }^{14}$ and aeroacoustic assessment ${ }^{15,16}$ are available as well. In the next section the assessed configurations are described. Then the aerodynamic performance and assessment are given followed by the aeroacoustic analysis. At the end a summary with lessons learned and some conclusions are provided.

\section{High-Lift Configurations and Flow Conditions}

Five high-lift landing configurations have been analyzed. The high-lift designs of these configurations were carried out within the Clean Sky GRA consortium in 2D assuming infinite wing sweep (2.5D). The underlying wing is designed for laminar flow (NLF) and hence the high-lift configurations are without a conventional forward slat. The 3D extension of the high-lift landing designs were also carried out inside the consortium and then provided to ALONOCO where they have been assessed. The wing has a wing kink at about $34 \%$ span where the flaps are divided in inner and outer parts. All configurations are without a tail and the wing with its high-lift devices are mounted on a fuselage.

Configuration 1 consists of a wing with a double slotted flap; this configuration is denoted as the baseline and is the configuration that was initially evaluated in the project. The other configurations represent further development of this configuration with improved performance in $2.5 \mathrm{D}$ and that have been evaluated and assessed in ALONOCO.

Configuration 2 consists of the same wing and a single slotted flap. Configurations 3 and $3 \mathrm{~b}$ are booth droopnose configurations where the wing droop deflection starts close to the fuselage and ends at about $67 \%$ span; the flap(s) ends further outboard at about $79 \%$ span. Configuration 3 has a droop that linearly decreases with increased span whereas Configuration $3 \mathrm{~b}$ has a constant droop span-wise. Configuration 4 has the same single slotted flap and a Krueger flap in front of the wing leading edge almost along the entire span. It is deployed from the lower side of the wing.

A three-dimensional picture of the baseline configuration with its double slotted flap is shown in Fig.

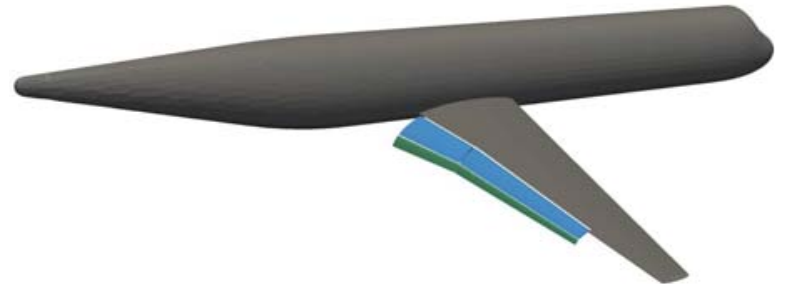

Figure 1. Configuration 1 with double slotted part span flap.

1. The wing has a trailing-edge kink and the high-lift system has separate elements in- and outboard of the kink which is the same for all configurations. Span wise cuts at an inboard position of all configurations are displayed in Figure 2. The wing has a moderate leading edge sweep and the wing total span is about $33.5 \mathrm{~m}$ with a total wing area of $108 \mathrm{~m}^{2}$. The mean aerodynamic chord is $3.6 \mathrm{~m}$ and the length of the fuselage is $37.8 \mathrm{~m}$.

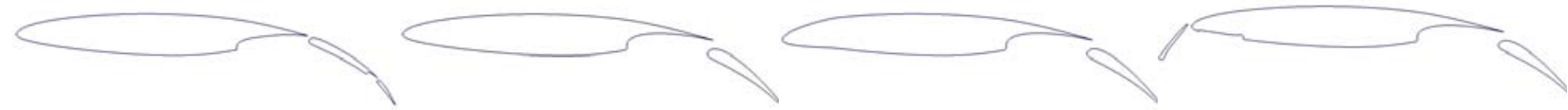

Figure 2 Span wise cuts of configurations 1-4 from left to right. Configuration 1 (baseline) - double slotted flap. Configuration 2 - single slotted flap. Configuration 3+3b-single slotted flap + droop nose. Configuration $4-$ single slotted flap + Krueger flap. 
All five configurations are analyzed for their aerodynamic performance. Configurations $1,2,4$ have been analyzed for their aeroacoustic performance. The flow conditions for all calculations are $\mathrm{M}_{\infty}=0.2$ and $\mathrm{Re}=17 \times 10^{6}$, where the Reynolds number is based on the mean aerodynamic chord. The aerodynamic calculations are performed up to and beyond maximum lift. The aeroacoustic calculations are carried out at an angle of attack that corresponds to a constant lift in the vicinity of the maximum lift obtained for configurations 1-3.

\section{Aerodynamic Assessment}

\section{A. Computational tool and settings}

The CFD solver employed in the calculations is the Edge code (http://www.edge.foi.se/), which is an edge- and node-based Navier-Stokes flow solver applicable for both structured and unstructured grids. ${ }^{17-20}$ Edge is based on a finite volume formulation where a median dual grid forms the control volumes with the unknowns allocated in the centres. The governing equations are integrated with a line-implicit approach ${ }^{19}$ in areas with highly stretched elements and explicitly elsewhere with a multistage Runge-Kutta scheme to steady state and with acceleration by FAS agglomeration multigrid. Weak boundary conditions are applied at all boundaries for all flow variables. ${ }^{20} \mathrm{~A}$ pre-processor creates the dual grid and the edge based data structure, which is also employed to agglomerate coarser control volumes for the multigrid and to split up the computational domain for parallel calculations. ${ }^{21}$ The present calculations employ a second-order central discretization of the convective terms with a small amount of artificial dissipation.

The aerodynamic assessment is based on calculations up to maximum lift, these calculations are steady state RANS (Reynolds Averaged Navier-Stokes) calculations with an Explicit Algebraic Reynolds Stress Models $(\mathrm{EARSM})^{22,23}$ to model the turbulence. In average, 5000 multigrid iterations were conducted to reach a steady state. Beyond maximum lift the convergence was poor with oscillations of moderate amplitude in the computed forces and moments. Although the wing at cruise conditions has a large part where the flow is laminar, the flow is assumed to be fully turbulent in all calculations for the high-lift configurations. The assumption is based on initial stability calculations for the baseline configuration with a data base method ${ }^{2,24}$ for which transition to turbulent flow was detected close to the stagnation points on both the wing and flaps.

\section{B. Computational grids}

The configurations were delivered as CAD files from Alenia to FOI, all meshing and calculations were carried out on half models with a symmetry plane through the fuselage. ICEM was used to generate initial inviscid unstructured grids. The in-house grid generator Tritet ${ }^{25,26}$ was then used to generate the hybrid unstructured grids with prismatic elements in the near wall region and tetrahedral elements further away. Typically 35 prismatic elements

Table 1 Size of computational grids for RANS calculations.

\begin{tabular}{lcc}
\hline Configuration & No. of nodes & No. of surface nodes \\
\hline Configuration 1 & $16.0 \times 10^{6}$ & $0.41 \times 10^{6}$ \\
Configuration 2 & $24.3 \times 10^{6}$ & $0.60 \times 10^{6}$ \\
Configuration 3 & $17.7 \times 10^{6}$ & $0.45 \times 10^{6}$ \\
Configuration 3b & $21.9 \times 10^{6}$ & $0.55 \times 10^{6}$ \\
Configuration 4 & $31.0 \times 10^{6}$ & $0.85 \times 10^{6}$ \\
\hline \hline
\end{tabular}

are used to obtain $\mathrm{y}^{+} \sim 1$ at the first inner node, the numbers of layers vary slightly and a few pyramidal elements are also used in the transition region between prismatic and tetrahedral cells. The total grid sizes vary between 16 and 31 million nodes as given in Table 1. Note that the grid for Configuration 4 is finer due to the additional forward Krueger flap.

The surface grid of details from the configurations can be seen in Fig. 3. The grids are clustered along the leading and trailing edges and at the tip of the wing and flap elements. 

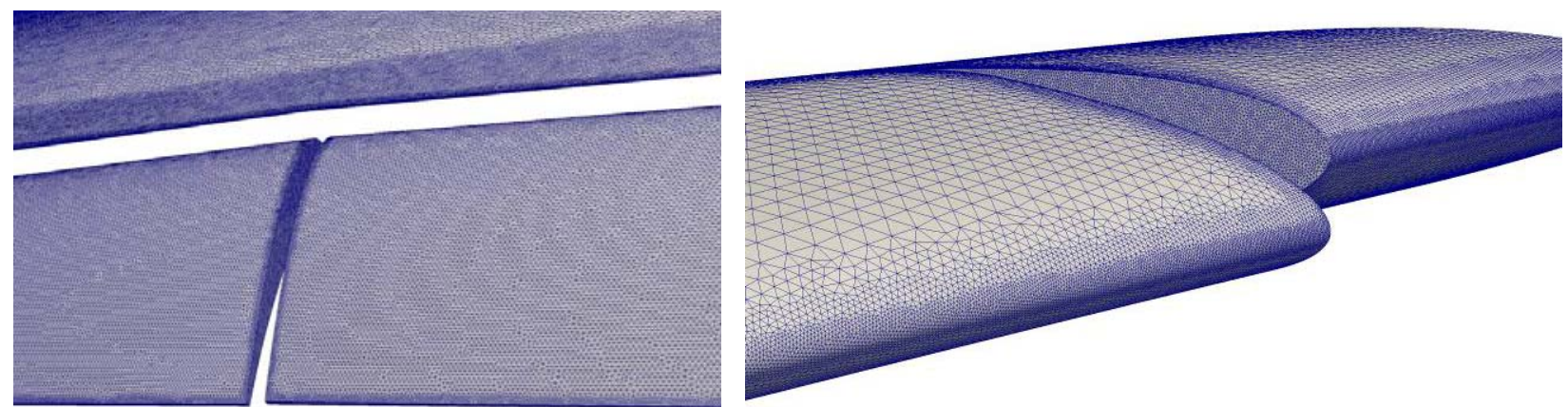

Figure 3 Surface grids for the aerodynamic analysis. Left: single slotted flap of Configurations 2-4 in the vicinity of the kink. Right; leading edge of Configuration $3 b$ at about $65 \%$ span where the constant droop ends.

\section{Computational results}

RANS calculations were carried out for all configurations with the purpose to analyze the aerodynamic performance for pre- and post-stall conditions. In the vicinity of maximum lift the flow computations were typically initiated with a solution from the closest lower incidence.

The computed lift $\left(\mathrm{C}_{\mathrm{L}}\right)$ and lift over drag $\left(\mathrm{C}_{\mathrm{L}} / \mathrm{C}_{\mathrm{D}}\right)$ polars are displayed in Fig. 4. Several interesting observations can be made. The first and most striking observation is that Configuration 4 is vastly superior to the other configurations in terms of maximum lift; the maximum lift is close to $20 \%$ higher for this configuration occurring at an incidence of 5-7 degrees higher than for the other configurations. The other configurations all have more or less the same maximum lift but at different angles of attack, Configuration 1 gives a higher lift at a lower incidence due to its double slotted flaps. Configuration 2,3,3b show very similar results in terms of both lift and drag ahead of maximum lift which is expected. More surprisingly though, is that the configuration with droop nose do not show any significant increase of maximum lift.

The highest efficiency in terms of $C_{L} / C_{D}$ is obtained for Configurations 2,3,3b before stall, the efficiency is the lowest for Configuration 1 and with Configuration 4 in between.. The reduced efficiency for Configuration 4 at lower incidences is due to the additional drag from the Krueger flap. Beyond stall for configurations 1-3b, the Configuration 4 is superior in every aspect up to its maximum lift.
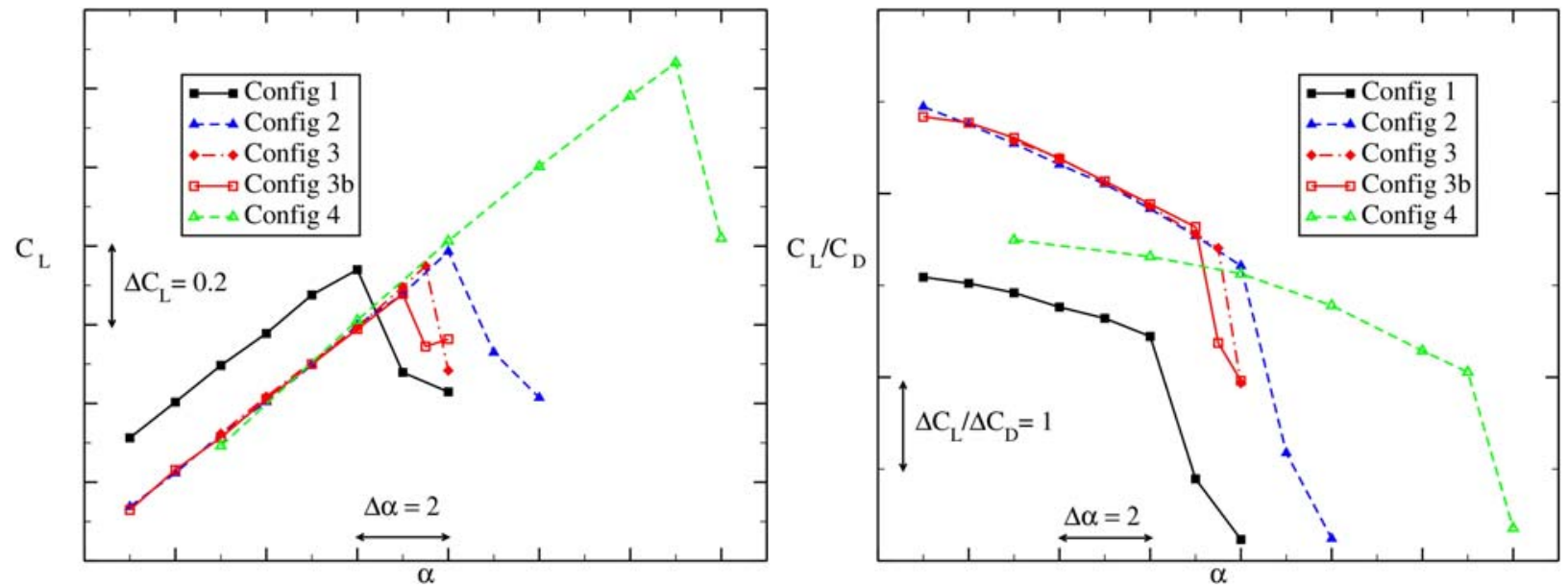

Figure 4. Aerodynamic coefficients. Left: $\operatorname{Lift}\left(C_{L}\right) v s . \alpha$. Right: $C_{L} / C_{D} v s . \alpha$.

To investigate how the flow breaks down beyond maximum lift the stream-wise components of the skin friction vector is plotted on the upper side at one degree beyond maximum lift in Fig. 5. It should be noted that the flow solutions are not entirely steady and hence there may be small fluctuations in the position and size of the separated areas. Configurations 1-3b all have a similar behavior with separation occurring on the wing at a mid or outer spanwise position. The separated areas are somewhat larger for configurations 1, 2 compared to $3,3 \mathrm{~b}$. It also seems that the constant droop in Configuration $3 \mathrm{~b}$ has shifted the separated area to a more outboard position and reduced its size slightly. Nevertheless, the flow separates at a span wise position just outboard of the end of the droop which explains why there is no gain from the droop of the nose. Note also that the flow over the flaps is mainly attached. 
The main difference in flow separation occurs for Configuration 4 with an entirely different separation pattern at an inboard position. The separation starts inboard of the Krueger flap at the wing leading edge and propagates and grows in size downstream to the trailing edge. The flow over the flap is still attached though. It is also worth to notice that the flow over the Krueger flap remains attached.

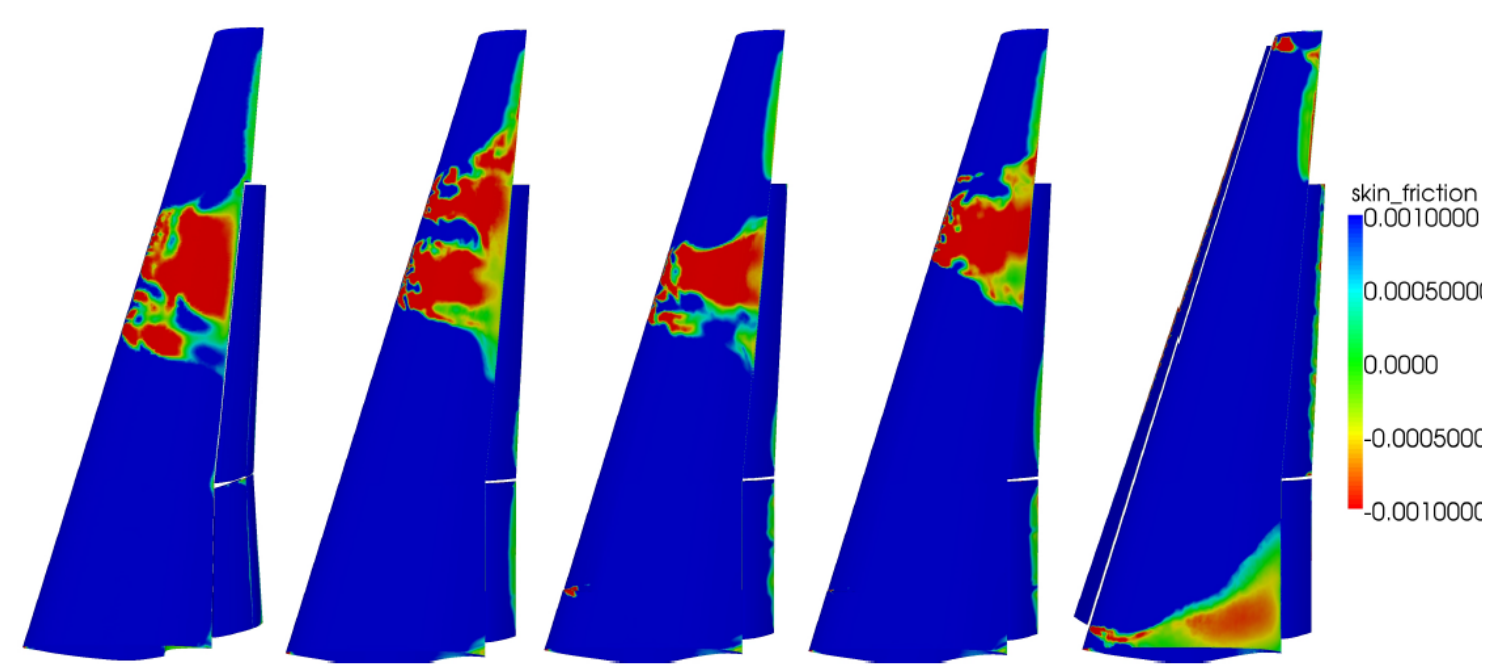

Figure 5. Plots of x-component of skin friction on upper side. From left to right: Configurations 1,2,3,3b,4. Angle of attack of about one degree beyond maximum lift.

\section{Aeroacoustic Assessment}

Since there was no gain in the aerodynamic performance from the two drooped nose configurations, it was decided that only three configurations, Configuration 1,2 and 4, were to be assessed for their aeroacoustic performance.

\section{Computation of the noise source}

The calculation of the noise source is based on turbulence resolved calculations of the configurations using the Edge CFD code as for the aerodynamic assessment described above. An algebraic (zero-equation) in-house hybrid RANS-LES model is used ${ }^{27}$ which has proven to be computationally efficient and accurate for various applications. ${ }^{9-11}$ The eddy viscosity is calculated from a mixing-length type model. In the near wall region where the model works in RANS mode, the length scale is given by a RANS length scale involving the wall distance. Further away from the wall, the model switches to LES mode and the eddy viscosity is evaluated in accordance with the Smagorinsky model.

The unsteady nature of hybrid RANS-LES computations implies a need for different computational settings compared to those of the RANS computation. The free stream turbulence level was set to correspond to $1 \%$ which is the same as for the RANS computations. All hybrid simulations were initiated with RANS solutions. A $2^{\text {nd }}$ order implicit backward difference scheme is used in time with dual time stepping in each physical time step with the same type of convergence acceleration (multigrid, line-implicit time integration) as in the steady RANS calculations above. Usually 60-100 dual multigrid cycles were required in each time step. The time step was chosen with the ambition to resolve well frequencies up to at least $1 \mathrm{kHz}, \Delta \mathrm{t}=5 \times 10^{-5}$, and 10,000 time steps were conducted corresponding to $0.5 \mathrm{~s}$ in physical time. The time step corresponds to a non-dimensional time step of $\Delta \mathrm{t} \mathrm{c}_{\infty} / \mathrm{L}=$ $4.7 \times 10^{-3}$, or $\Delta t \mathrm{U}_{\infty} / \mathrm{L}=9.5 \times 10^{-3}$, where $\mathrm{c}_{\infty}, \mathrm{U}_{\infty}$, $\mathrm{L}$, denotes the speed of sound, free stream velocity and mean aerodynamic chord respectively. The frequency $1 \mathrm{kHz}$ corresponds to a Strouhal number of about $\mathrm{St}=50$ where the Strouhal number is defined as $\mathrm{St}=\mathrm{fL} / \mathrm{U}_{\infty}$. The first 2000 iterations are excluded in the aeroacoustic analysis below to allow for the flow to develop.

All calculations were carried out at a constant lift corresponding approximately to conditions close to maximum lift of configurations 1-2 in Fig. 4 above. This implies that Configuration 4 is computed at a flow condition far away from its maximum lift. Unsteady data is recorded and saved on the solid walls at each time step. In addition, some of 
the acoustic analogy methods described below require unsteady data on a surface enclosing the wall which is reflected in the grid generation described below.

\section{E. Computational grids}

The same geometry descriptions were used to generate grids for the hybrid RANS-LES calculations and half models were used for these calculations as well. New grids had to be generated since these calculations imply additional requirements on the mesh resolution and on data recording.

An integral surface was introduced in the computational grid on which unsteady data is retrieved and stored. The surface is the Kirchhoff surface and is treated as a transparent surface in the flow solver which implies that it has no influence on the computed results, but only used for data sampling and recording. The location of this surface has to be defined when generating the grids and the grid resolution has to be fine enough between the solid wall and this surface to resolve the necessary scales. With the ambition to spatially resolve frequencies well up to $\mathrm{St}=50$ and with 20 cells per wave length, a maximum cell size of about $1,5 \mathrm{~cm}$ was respected in areas where the main part of the sound is expected to be generated, i.e. in bay areas, around the flaps and the Krueger flap.

To satisfy these requirements it is hence of utmost importance that the Kirchhoff surface is placed at an optimal position. If the boundary is too far away the grid will become too large for affordable calculations. If it is too close with wakes and boundary layers intersecting the sampling boundary, on the other hand, it will have a negative effect on the accuracy of the integrated far-field noise. The approach chosen for the selection of the location of the Kirchhoff boundary is based on the magnitude of the vorticity from the corresponding RANS solution. The location of the Kirchhoff surface has been selected based on the vorticity magnitude such that the surface encloses all regions with high levels of vorticity.

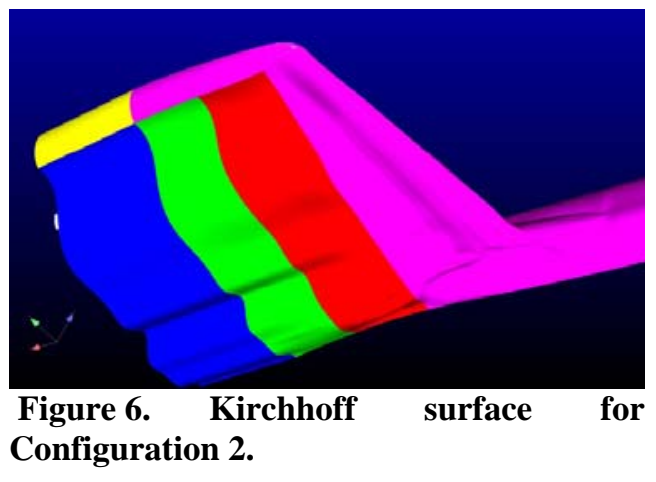

The Kirchhoff surface for one of the configurations is displayed in Fig. 6 where it can be seen that the surface extends to a position down stream of the end of the fuselage. The sections of a RANS and RANS-LES grid are displayed in Fig. 7 where the Kirchhoff surface is located in the region where the mesh refinement ends and is indicated with a different color. The sizes of the meshes for the aeroacoustic analysis are given in Table 2 . The grid size vary between 24 and 44 million nodes and are between $24-40 \%$ finer in terms of total number of grid nodes and $5-20 \%$ finer in terms of boundary nodes. The Kirchhoff surfaces contain about $0.25-0.37 \times 10^{6}$ nodes. More than $90 \%$ of all nodes are located in the Table 2 Grid sizes for RANS-LES calculations. region between the aircraft and the Kirchhoff surfaces.

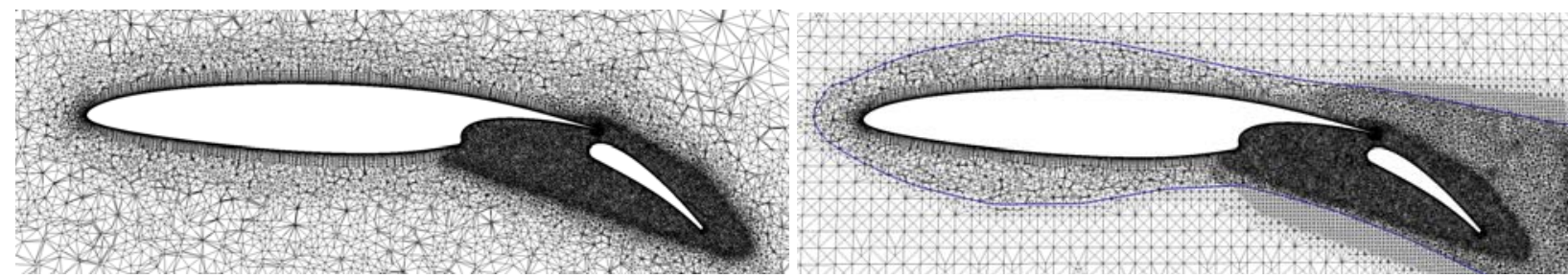

Figure 7. Inboard span-wise section of RANS (left) and hybrid RANS-LES grid (right) for Configuration 2.

\section{F. Acoustic analogy methods}

The aeroacoustic performance is assessed for configurations 1,2, 4 with three different acoustic analogy methods; the methods are based on integrating the unsteady near-field data from the recorded data from the RANS- 
LES calculations and propagated to far-field observers. The three

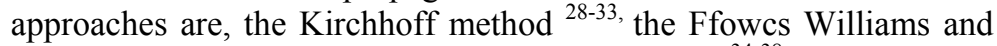
Hawkings (FWH) method for permeable surface ${ }^{34-38}$ and the Curle method ${ }^{39-41}$.

The three methods are based on surface integral over the aircraft configuration and/or the Kirchhoff surface. The applications of these methods in the assessment of different configurations, as well as the comparison of these three methods are reported by Yao et al. ${ }^{15-16}$. The Curle approach provides in general lower noise levels than the other two approaches since only fluctuations on solid wall surfaces are accounted for. The advantage with the Curle approach is that it is possible to indicate the contribution of different aircraft components to the far-field noise level. The Ffowcs Williams and Hawkings is the approach that is considered the most accurate in general.

The acoustic analogy methods propagate the noise to far-field observers located $500 \mathrm{~m}$ from the aircraft. The observers are positioned in the plane of symmetry according to Fig. 8. Following the

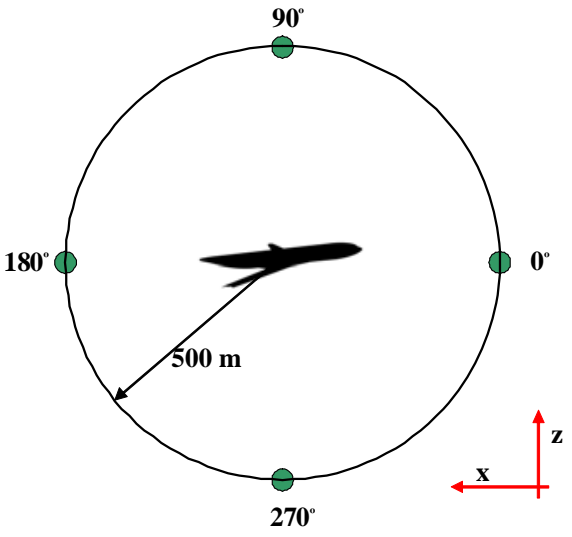

Figure 8. Position of far field observes in relation to the aircraft noise source. notation Fig. 8 an angle of $0^{\circ}$ means that the observer is located at a position $500 \mathrm{~m}$ in front of the aircraft (the aircraft is approaching the observer), $270^{\circ}$ is a position below the aircraft and $180^{\circ}$ is a position behind the aircraft.

\section{G. Numerical results}

Figure 9 illustrates the r.m.s. of pressure fluctuations on the lower side of the three configurations. The largest fluctuations occur in the main wing cove and on the flaps. The fluctuations are larger at inboard stations, in particular on the flaps. It can also be seen that the fluctuations are larger on the double slotted flaps of Configuration 1 compared to the other two configurations; the fluctuations are also higher on the inboard part of the wing. Also worth noticing is that the fluctuations on the forward Krueger flap are small.

In Fig. 10 the sound pressure levels (SPL) below the aircraft are plotted as a function of the frequency for the three analyzed configurations using the Curle method, where the SPL due to different components is plotted and so is the total SPL in the downward direction. The corresponding plots of the overall sound pressure levels (OASPL) are further displayed for the three configurations in Fig. 11 in which the directivity is plotted. The Curle approach allows to
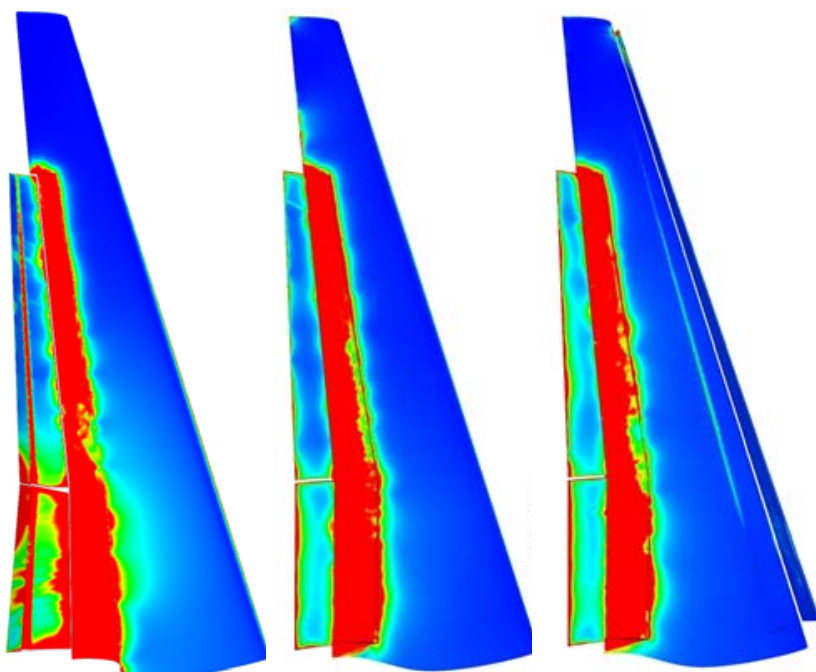

Figure 9. Pressure fluctuations on the lower side. From left to right: Configurations 1,2, 4.

distinguish the contribution from different parts of the aircraft since it only involves integration over the solid surface. The SPL in Fig. 10 is given as a function of the non-dimensional frequency, or Strouhal number, in the range between $0.5 \leq \mathrm{St} \leq 50$ which corresponds to a frequency range of $10-1 \mathrm{kHz}$. The SPL has a fairly flat appearance for lower frequencies indicating broadband noise without any significant tonal signals. The SPL is reduced at higher frequencies for $\mathrm{St} \geq 5$.

The plots of SPL and OASPL for Configuration 1 contain separate plots for the wing, the wing cove, the front (forward) flap divided in an inner and outer part of the kink, the cove of the front inner and outer flap and the rear (back) flap divided in an inner and outer part. The main contribution comes from the wing, the wings cove and the inner part of the forward (front) flap. The plot for Configuration 2 contains noise contributions from the wing, the wing cove, the inner and outer part of the flap. The major contribution comes from the inner part of the flap. The total noise is in general lower than that for Configuration 1. The plot for Configuration 4 is divided in the same components as for Configuration 2 with the addition of the contribution from the Krueger flap. The inner part of the flap is still the dominating noise source and the contribution from the Krueger flap is considerably smaller than for the other components. The OASPL for Configuration 4 is somewhat higher than that from Configuration 2, and is similar to the levels of Configuration 1. Since the only geometric difference between Configuration 1 and 4 is the 
Krueger flap that does not contribute significantly to the noise, one would expect similar noise levels. A possible explanation to the observed difference could be due to the fact that the simulation for Configuration 4 is carried out at a flow condition about $20 \%$ from its maximum lift whereas for Configuration 2 the simulations occur close to its maximum lift.
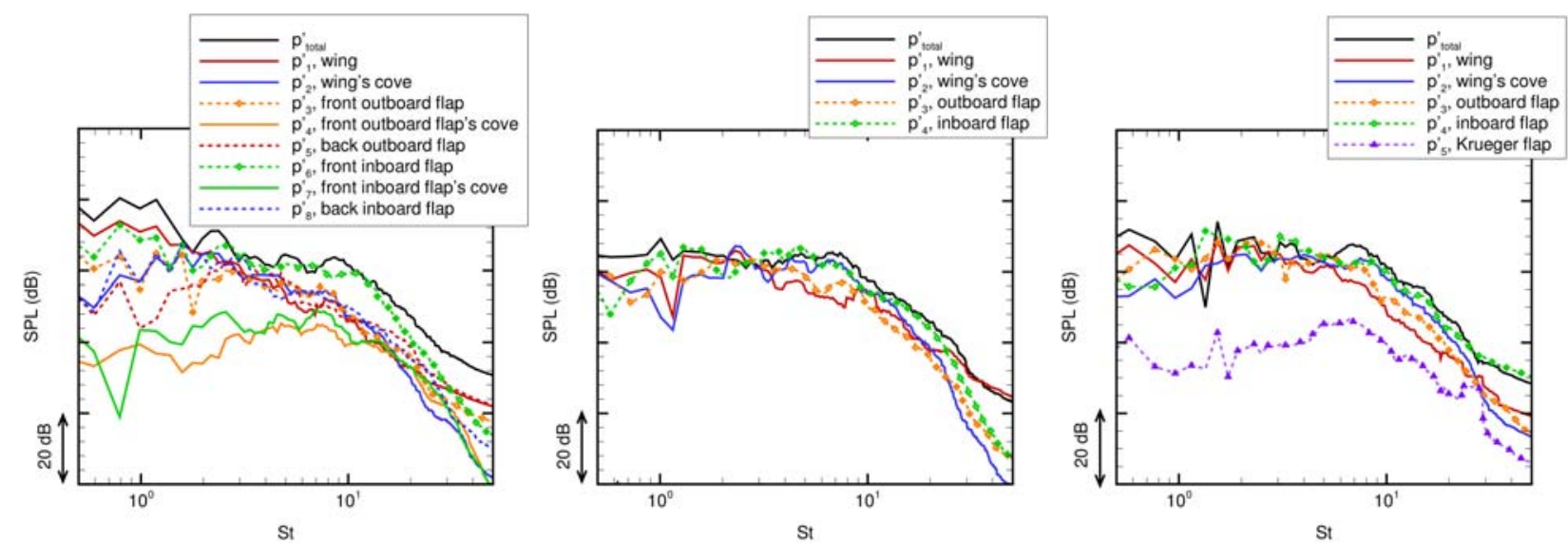

Figure 10. 1/3 octave smoothed SPL in downward direction $\left(270^{\circ}\right)$ from analysis with the Curle method From left to right: Configurations 1,2 and 4 .
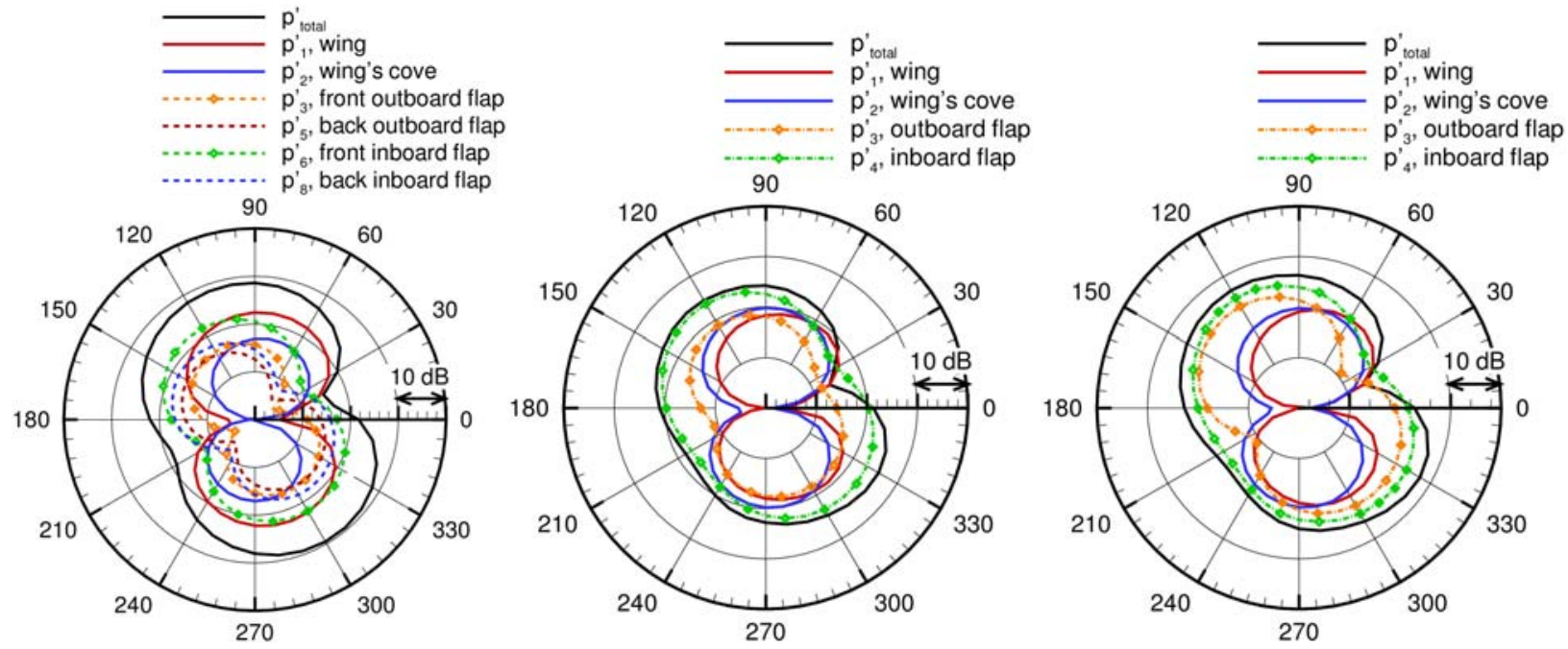

Figure 11. OASPL from analysis with the Curle method. From left to right: Configurations 1, 2 and 4.

Figures 12 and 13 compare the SPL (downward direction) and OASPL obtained for the three configurations with the three acoustic analogy methods. The SPL is in general higher for the baseline Configuration 1 and the lowest for Configuration 4 which is most noticeable at lower frequencies. The noise levels are 6-10 dB lower for Configuration 4 compared to the other configurations. The predicted noise levels with the Curle method are in general lower than from the two other approaches

The levels of SPL and OASPL are relatively similar between the Kirchhoff and FWH methods although Configuration 1 gives higher SPL at lower frequencies. The most noticeable difference between the two methods occurs for Configuration 2, where higher noise levels are predicted with the Kirchhoff method, particularly in the forward direction $\left(0^{\circ}\right)$. Further analysis of the terms in the integrands over the Kirchhoff surfaces shows that the main contribution to the noise comes from the time derivative term for the Kirchhoff method and from the normal pressure derivative in the FWH method. Furthermore, the major difference in the noise levels occurs at low frequencies, as is verified in Fig. 14, where the corresponding OASPL is plotted for the configurations using the Kirchhoff method and accounting only for frequencies $\mathrm{St} \geq 1$. Similar levels for OASPL are obtained for all configurations, but the levels are lower due to the exclusion of the lowest frequencies. 

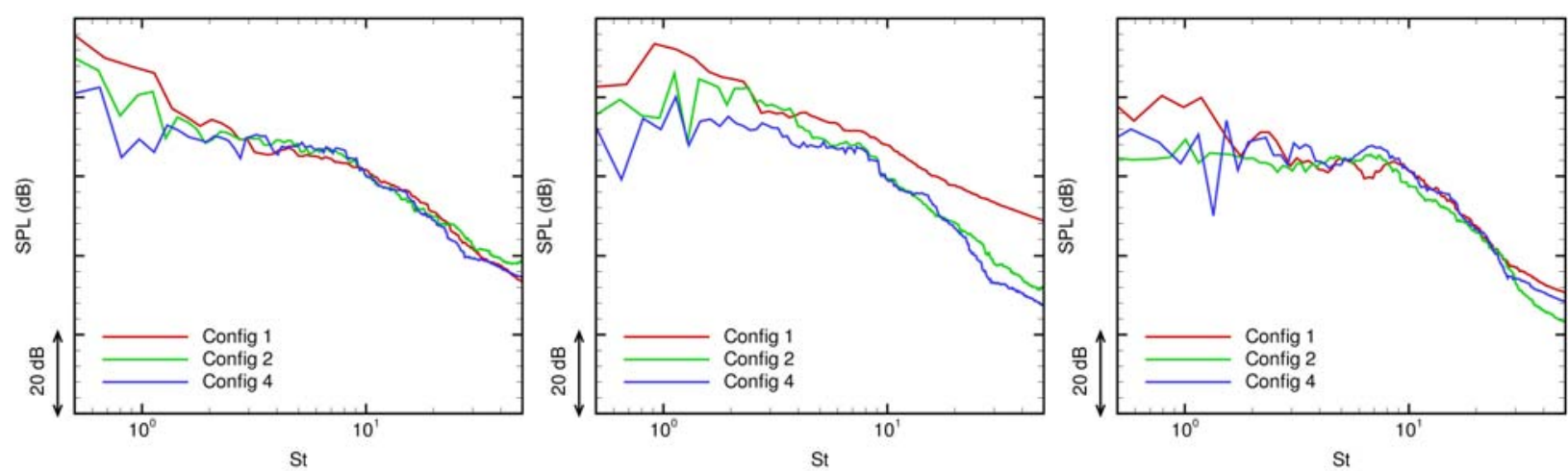

Figure 12. 1/3 octave smoothed SPL in downward direction $\left(270^{\circ}\right)$ for Configurations 1,2 , 4 . From left to right: Kirchhoff method, Ffowcs Williams and Hawkings (FWH) method, Curle method.

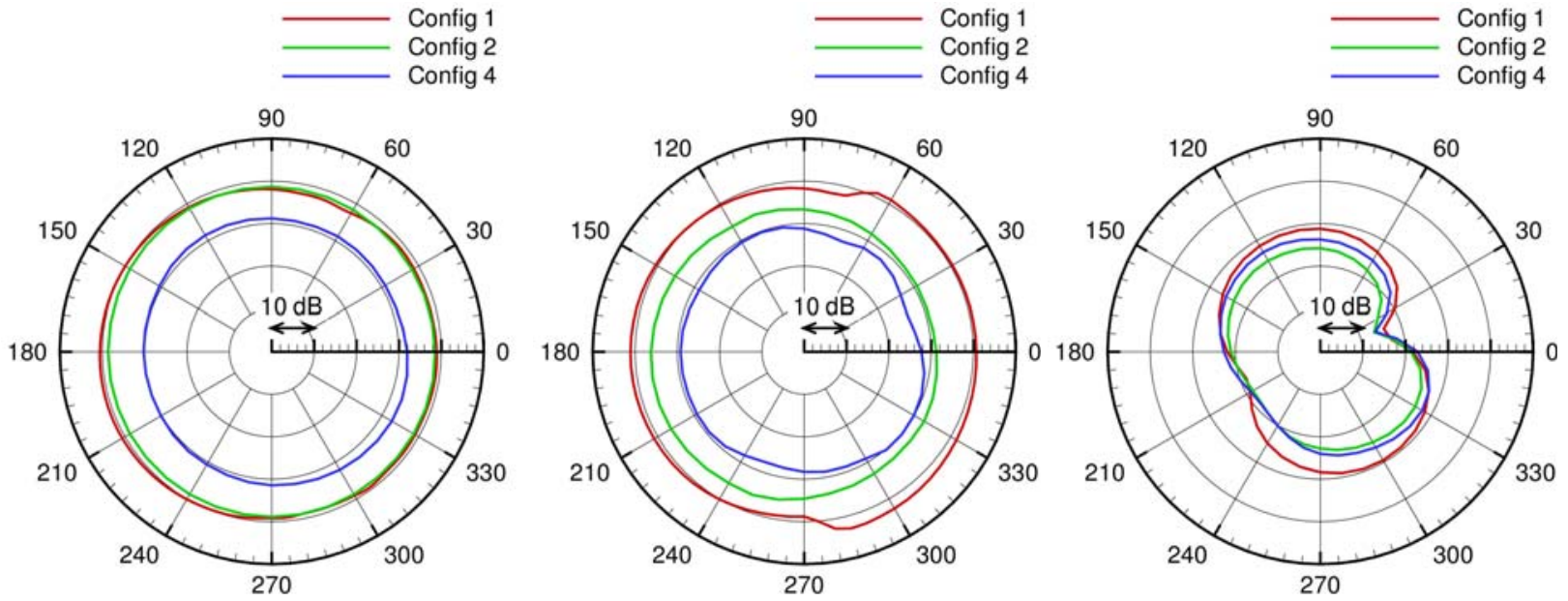

Figure 13. OASPL for Configurations 1, 2, 4. From left to right: Kirchhoff method, Ffowcs Williams and Hawkings (FWH) method, Curle method.

In Fig. 15 the contribution to the OASPL predicted by the Curle method from different aircraft components are displayed, the components being the wing without cove, the wing cove and the inner (kink inboard) part of the flap (two flaps for Configuration 1). The contribution from the wing is higher with Configuration 1 and about similar with the two other configurations. The contribution from the wing cove, however, is smaller for Configuration 1 where higher frequencies have a lower SPL values. The highest noise levels are obtained for the inboard flap(s) for which all configurations obtain similar levels. Hence, the main contributing factor to the reduced noise of Configuration 4 is the reduction of noise over the wing.

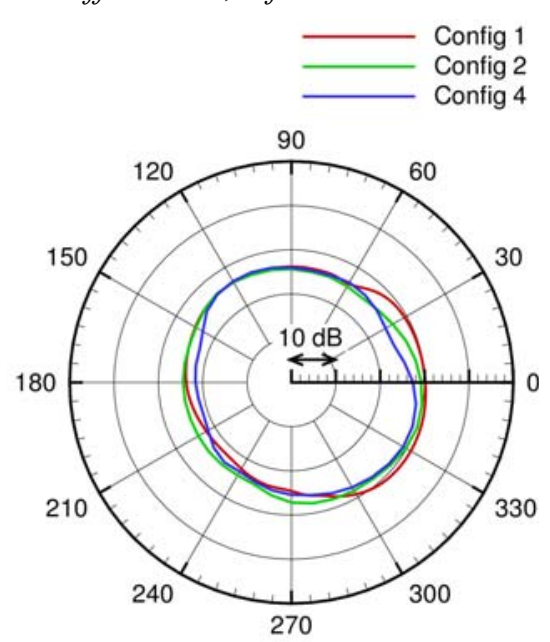

Figure 14. OASPL for Configurations 1, 2, 4; Kirchhoff method. Frequencies $S t \geq 1$. 

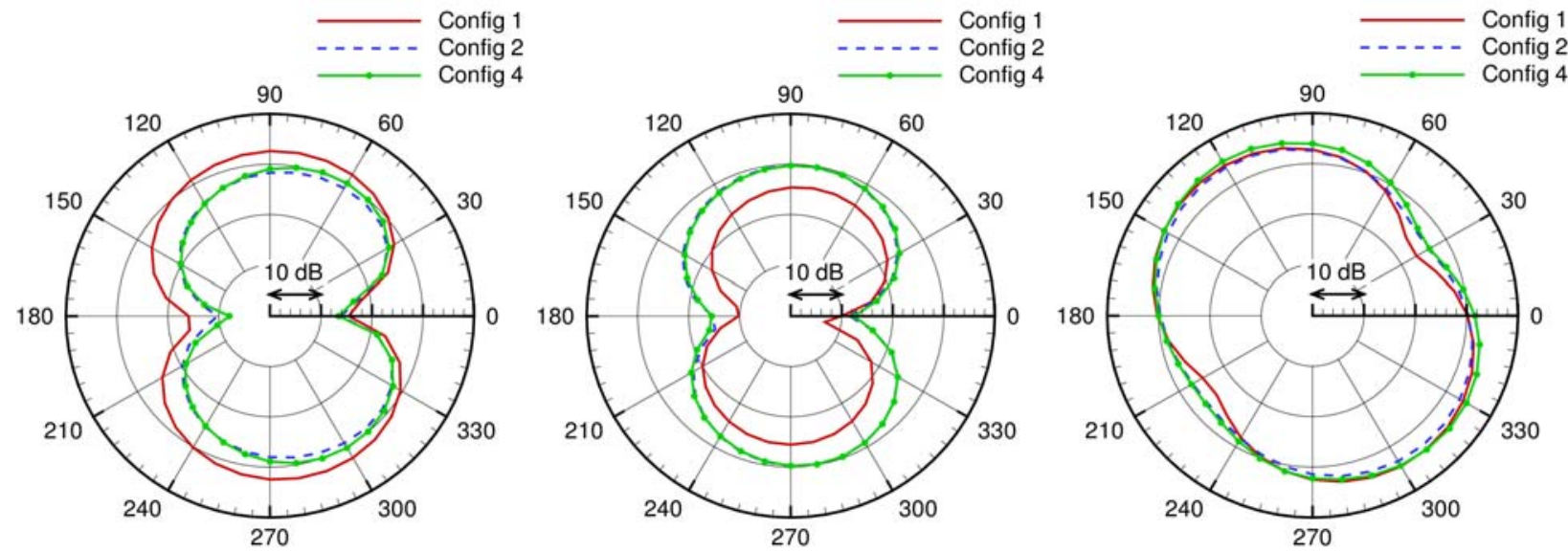

Figure 15. OASPL from analysis with the Curle method for Configurations 1, 2, 4. From left to right: contribution from the wing without cove, from the wing cove and from the inner (inboard kink) flap(s).

\section{Summary and Conclusions}

The paper presents a summary of the aerodynamic and aeroacoustic assessment of high-lift configurations for a laminar wing of a regional aircraft. The configurations assessed consist of one baseline and four low-noise configurations. The slat-less high-lift configurations involve a double slotted flap baseline configuration (Configuration 1), a single slotted flap configuration (Configuration 2), two drooped nose configurations with single slotted flap (Configuration 3, 3b) and a configuration with a forward Krueger flap and single slotted flap (Configuration 4). The aerodynamic analysis was carried out by steady RANS calculations up to maximum lift. The aeroacoustic analysis is based on turbulence-resolving simulations using a hybrid RANS-LES model at flow conditions corresponding to a constant lift for each configuration. Unsteady data sampled from these calculations are used as inputs in three acoustic analogy methods that have been used to analyze far-field noise propagation. The sampling of unsteady data has been taken on the aircraft surface and on a Kirchhoff surface enclosing the most potent noise-generating flow regions. High grid resolution is patched cross the wall boundary layer and up to the Kirchhoff surface. The location of Kirchhoff surface has been optimized in such a way that it encloses the significant flow-induced noise sources for accurate analysis but is not too far away from the wall boundary layer for affordable computations. The integral surface has been determined by referring to the magnitude of vorticity.

For the aerodynamic analysis, hybrid unstructured grids were generated. The aerodynamic investigation shows that the configuration with a Krueger flap is the best performing high-lift system. Although the efficiency $\left(C_{L} / C_{D}\right)$ is slightly lower than for the other single slotted flap configurations at lower incidences, the maximum lift is almost $20 \%$ higher compared to other configurations. The droop of the nose has no significant effect on the maximum lift since the onset of separation starts outboard span of the droop. In addition, the stall characteristic for the Krueger flap configuration is different and more favorable with a span-wise inboard type of separation whereas all the other configurations show separation at an outboard station.

For the aeroacoustic analysis a set of refined grids were generated and the Kirchoff surface was incurred. The grids contain up to 45 million nodes. Time dependent data is used as inputs to three acoustic analogy methods, namely, the Curle, the Kirchhoff and the Ffowcs Williams and Hawkings (FWH) method. These methods are applied to all the configurations and a comparative study has been conducted. It is shown that the configuration with a Krueger flap produces the best aeroacoustic performance. The perceived noise level from this configuration of an observer below the aircraft is $6-10 \mathrm{~dB}$ lower compared to the other configurations. The reduced noise level is mainly due to the reduction of noise intensities at loweer frequencies. The dominant noise source with all configurations is the inboard part of the flap. The noise induced by the Krueger flap is insignificant. As expected, the Curle method predicts lower noise levels as compared to the Kirchhoff and FWH methods.

Due to the lack of experimental testing for these conceptual new low-noise high-lift configurations, it is not possible to conduct experimental validation at this stage. Nevertheless, it is believed that the computations are of a good quality based on our previous experience. Moreover, it should be noted that the main purpose of the work was to evaluate and assess these conceptual configurations. The predicted levels of aerodynamic and aeroacoustic quantities are of interest in the studies, but the most important concern is on the difference (both in predicted aerodynamic and aero-acoustic properties) between the configurations for a relevant assessment. The required grid 
resolution is very high. It is noted here that an improved "academic" aero-acoustic analysis for the configurations studied in this work would have to invoke grids being refined much beyond the current resolution. The present work should be viewed more of an engineering study. Some of the difference observed in the aeroacoustic analysis may be explained by insufficient grid resolution. Nevertheless, the noise levels are realistic and the difference between the configurations is believed to be correct by which the assessment has been made relevant.

\section{Acknowledgement}

The work was partly supported by the Clean Sky Joint Undertaking (CSJU) under contract No. CS-GA-2009255714. The project was monitored by Dr. Michele Averardo at Alenia Aeronautica.

\section{References}

${ }^{1}$ Seitz, A., Kruse, M., Wunderlich, T. "The DLR Project LamAir,: Design of a NLF Forward Swept Wing for Short and Medium Range Transport Aircraft", AIAA paper 2011-3526, 2011.

${ }^{2}$ Perraud, J., Arnal, D., Casalis, G., Archambaud, J.-P. "Automatic Transition Predictions Using Simplified Methods", AIAA Journal, Vol. 47, No. 11, 2009.

${ }^{3}$ Macaraeg, M.G., "Fundamental Investigations of Airframe Noise", Fourth AIAA/CEAS Aeroacoustic Conference, France, AIAA Paper 98-2224, 1998.

${ }^{4}$ Crighton, D. G. "Airframe Noise", in Aeroacoustics of Flight Vehicles: Theory and Practice, Volume I: Noise Sources, NASA Reference Publication 1258, WRDC Technical Report 90-3052. H. H. Hubbard, ed., 1991, pp. 391-447.

${ }^{5}$ Ewert, R., "Broadband Slat Noise Prediction Based on CAA and Stochastic Sound Sources from a Fast Random Particle Mesh (RPM) Method," Computers and Fluids, Vol. 37, 2008, pp. 369-387.

${ }^{6}$ Lockard, D. P. and Choudhari, M. M., "Noise Radiation from a Leading-Edge Slat," 15th AIAA/CEAS Aeroacoustics Conference, AIAA 2009-3101, 2009.

${ }^{7}$ Peng, S.-H., Simulation of turbulent flow past a rectangular open cavity using DES and unsteady RANS. AIAA Paper 20062827, 2006.

${ }^{8}$ Peng, S.-H. and Leicher, S., 2008. DES and Hybrid RANS-LES modelling of unsteady pressure oscillations and flow features in a rectangular cavity. In Peng, S.-H and Haase, W. (Eds) "Advances in Hybrid RANS-LES Modelling", Notes on Numerical Fluid Mechanics and Multidisciplinary Design, Springer, Vol. 97, pp.132-141.

${ }^{9}$ Peng, S.-H., Eliasson, P. "Unsteady Simulation for the Flow Over a Three-Element High-Lift Configuration at Stall”, AIAA 2008-4143.

${ }^{10}$ Nebenführ, B., Peng, S.-H, Davidson, L., "Hybrid RANS-LES Simulation of Turbulent High-Lift Flow in Relation to Noise Generation", 4th Symposium on Hybrid RANS-LES Methods, Beijing, 28-30 September, 2011.

${ }^{11}$ Spalart, P. R.,, Shur, M., Strelets, M. and Travin, A. K., "Initial noise predictions for rudimentary landing gear", Journal of Sound and Vibration, Vol. 330 , pp. 4180-4195, 2011.

${ }^{12}$ Deck, S. "Zonal-Detached-Eddy Simulations of the Flow around a High-Lift Configuration", AIAA Journal, Vol. 43, No. 11, 2005, pp. 2372-2384.

${ }^{13}$ Rakshani, B., Filippone, A. "Large-Eddy Simulation for Aerodynamic Noise from High-Lift Device”, AIAA Paper 20090281, 2009.

${ }^{14}$ Grundestam, O., Peng, S.-H., Eliasson, P., Yao, H.-D., Davidson, L., Eriksson, L.-E. "Local Flow Properties in Relation to Noise Generation for Low-Noise High-Lift Configurations", AIAA Paper, 50th-Aerospace Science Meeting, 2012.

${ }^{15}$ Yao, H.-D., Davidson, L., Eriksson, L.-E., Grundestam, O., Peng, S.-H., Eliasson, P. “Aeroacoustic Assessment of Conceptual Low-Noise High-Lift Wing Configurations”, AIAA Paper, 50th-Aerospace Science Meeting, 2012.

${ }^{16}$ Yao, H.-D., Davidson, L., Eriksson, L.-E., Grundestam, O., Peng, S.-H., Eliasson, P. "Surface Integral Analogy Approaches to Compute Noise Generated by 3D High-Lift Wing Configuration", AIAA Paper, 50th-Aerospace Science Meeting, 2012.

${ }^{17}$ Eliasson, P., "EDGE, a Navier-Stokes Solver for Unstructured Grids", Proceedings to Finite Volumes for Complex Applications III, 2002, pp. 527-534.

${ }^{18}$ Eliasson, P., Weinerfelt, P., "Recent Applications of the Flow Solver Edge", Proceedings to 7th Asian CFD Conference, Bangalore, India, 2007.

${ }^{19}$ Eliasson, P., Weinerfelt, P., Nordström, J. “Application of a Line-implicit Scheme on Stretched Unstructured Grids”, AIAA Paper 2009-0163.

${ }^{20}$ Eliasson, P., Eriksson, S., Nordström, J. "The Influence of Weak and Strong Wall Boundary Conditions on the Convergence to Steady-State of the Navier-Stokes Equations", AIAA Paper 2009-3551.

${ }^{21}$ Berglind, T., "An Agglomeration Algorithm for Navier-Stokes Grids", AIAA Paper 2000-2254, 2000.

${ }^{22}$ Wallin, S., Johansson, A. V., “An explicit algebraic Reynolds stress model for incompressible and compressible turbulent flows," Journal of Fluid Mechanics, Vol. 403, 2000, pp. 89-132.

${ }^{23}$ Hellsten, A., "New Advanced k- $\omega$ Turbulence Model for High Lift Aerodynamics," AIAA Journal, Vol. 43, No. 9, 2005, pp. 1857-1869.

${ }^{24}$ Eliasson, P., Hanifi, A., Peng, S.-H. "Influence of Transition on High-Lift Prediction for the NASA Trap Wing Model", AIAA Paper 2011-3009. 
${ }^{25}$ Tysell, L., “An Advancing Front Grid Generation System for 3D Unstructured Grids" Proceedings of the 19th ICAS Congress, Anaheim, California, USA, 1994, pp. 1552-1564.

${ }^{26}$ Tysell, L., "Hybrid Grid Generation for Complex 3D Geometries," Numerical Grid Generation in Computational Field Simulation, Proceedings of the 7th Conference, Whistler, British Columbia, Canada, Sept. 25-28, 2000, pp. 337-346.

${ }^{27}$ Peng, S.-H., "Hybrid RANS-LES modelling based on zero- and one-equation models for turbulent flow simulation." In Proceedings of 4th Int. Symp. Turb. and Shear flow Phenomena, Vol. 3, 2005, pages 1159-1164.

${ }^{28}$ Farassat, F., Myers, M. K. "Extension of Kirchhoff's Formula to Radiation from Moving Surfaces," Journal of Sound and Vibration, Vol. 123, No. 3, 1988, pp. 451-461.

${ }^{29}$ Lyrintzis, A "Review: The use of Kirchhoff's method in computational aeroacousics", ASME: Journal of Fluids Engineering, 116, 1994, pp. 665-676.

${ }^{30}$ Farassat, F. , "Acoustic Radiation from Rotating Blades - the Kirchhoff Method in Aeroacoustics," Journal of Sound and Vibration, Vol. 239, No. 4, 2001, pp. 785-800.

${ }^{31}$ Kirhhoff, G. R. "Zur Theorie der Lichtstrahlen,” Annalen der Physik und Chemie, Vol. 18, 1883, pp. $663-695$.

${ }^{32}$ George, A. R., Lyrintzis, A. S. "Mid-Field and Far-Field Calculations of Transonic Blade-Vortex Interactions," AIAA paper 86-1854, 1986.

${ }^{33}$ Freund, J. B., Lele S., Moin, P. "Calculation of the radiated sound field using an open Kirchhoff surface", AIAA Journal, Vol. 34, No. 5, 1996, pp. 909-916.

${ }^{34}$ di Francescantonio, P. "A New Kirchhoff Formulation for Transonic Rotor Noise," Journal of Sound and Vibration, Vol. 202, No. 4, 1997, pp. 491-509.

${ }^{35}$ Brentner, K. S., Farassat, F. "An Analytical Comparison of the Acoustic Analogy and Kirchhoff Formulation for Moving Surfaces,” AIAA Journal, Vol. 36, No. 8, 1998, pp. 1379-1386.

${ }^{36}$ Brentner K. S., Farassat, F. "Modeling Aerodynamically Generated Sound of Helicopter Rotors," Progress in Aerospace Sciences, Vol. 39, 2003, pp. 83-120.

${ }^{37}$ Lockard, D. P., Choudhari, M. M. "Noise Radiation from a Leading-Edge Slat," AIAA Paper 2009-3101, 2009.

${ }^{38}$ J. E. Ffowcs Williams and D. L. Hawkins, "Sound Generation by Turbulence and Surfaces in Arbitrary Motion," Philos. Trans. Roy. Soc., Vol. A 264, No. 1151, 1969, pp. 321-342.

${ }^{39}$ Curle, J. (1955) "The influence of solid boundaries upon aerodynamic sound", Proc. Roy. Soc., A 231, pp. 505-514.

${ }^{40}$ Goldstein, M. E. , editor, Aeroacoustics, McGraw-Hill Book Company, New York, 1976.

${ }^{41}$ Wagner, C., Thomas, H., Sagaut, P.“Large-Eddy Simulation for Acoustics,” Cambridge University, 2007. 